\title{
Antitumor Agents 281. Design, Synthesis, and Biological Activity of Substituted 4-Amino-7,8,9,10-tetrahydro-2H- benzo[ $h]$ chromen-2-one Analogs (ATBO) as Potent In Vitro Anticancer Agents
}

\author{
Yizhou Donga ${ }^{a}$, Kyoko Nakagawa-Goto ${ }^{a}$, Chin-Yu Laia , Susan L. Morris-Natschke ${ }^{a}$, \\ Kenneth F. Bastow ${ }^{b}$, and Kuo-Hsiung Lee ${ }^{a, c},{ }^{*}$ \\ a Natural Products Research Laboratories, UNC Eshelman School of Pharmacy, University of \\ North Carolina, Chapel Hill, North Carolina 27599-7568 \\ b Division of Medicinal Chemistry and Natural Products, Eshelman School of Pharmacy, \\ University of North Carolina, Chapel Hill, North Carolina 27599-7568 \\ ${ }^{c}$ Chinese Medicine Research and Development Center, China Medical University, Taichung, \\ Taiwan
}

\section{Abstract}

In our exploration of new biologically active chemical entities, we designed and synthesized a novel class of antitumor agents, substituted 4-amino-7,8,9,10-tetrahydro- $2 H$-benzo[ $h]$ chromen-2one (ATBO) analogs. We evaluated their cytotoxic activity against seven human tumor cell lines from different tissues, and established preliminary structure-activity relationships (SAR). All analogues, except $\mathbf{8 , 9}$, and $\mathbf{2 5 - 2 7}$, displayed potent tumor cell growth inhibitory activity. Especially, compounds $\mathbf{1 5}$ and $\mathbf{3 3}$ with a 4-methoxyphenyl group at position C-4 were extremely potent with $\mathrm{ED}_{50}$ values of 0.008-0.064 $\mu \mathrm{M}$ and $0.035-0.32 \mu \mathrm{M}$, respectively. Compound $\mathbf{1 5}$ was the most potent analog compared with structurally related neo-tanshinlactone (e.g., 1) and 4amino-2H-benzo[ $h]$ chromen-2-one (ABO, e.g., 4) analogs, and thus merits further exploration as an anti-cancer drug candidate.

\section{Keywords}

4-Amino-7,8,9,10-tetrahydro-2H-benzo[ $h]$ chromen-2-ones (ATBO); Cytotoxic activity; Neotanshinlactone

\begin{abstract}
One of the most challenging areas of research in both industry and academia is the discovery and development of new medicines.1, 2 In 2009, 103 new drugs were approved by the FDA's Center for Drug Evaluation and Research (CDER). ${ }^{3}$ Nineteen new molecular entities (NMEs) and six biologics license applications (BLAs) were filed among these approvals. 3 The pharmaceutical industry's demand for new leads with new scaffolds has never been
\end{abstract}

(C) 2010 Elsevier Ltd. All rights reserved.

“Corresponding author. Tel: 919-962-0066; fax: 919-966-3893; khlee@unc.edu.

Publisher's Disclaimer: This is a PDF file of an unedited manuscript that has been accepted for publication. As a service to our customers we are providing this early version of the manuscript. The manuscript will undergo copyediting, typesetting, and review of the resulting proof before it is published in its final citable form. Please note that during the production process errors may be discovered which could affect the content, and all legal disclaimers that apply to the journal pertain. 
greater.4 These facts prompted us to design and discover new biologically active chemical entities.

Previously, our group successfully developed new cytotoxic chemical entities, including neotanshinlactone (1, Figure 1) and its 4-ethyl analog 2,5, 6 for treating breast cancer, and these compounds are now in extended preclinical study. Structural simplification and optimization is a powerful tool for analog design and lead exploration. For example, this strategy was applied to the natural product halichondrin $\mathrm{B}$, a potent mitotic inhibitor, which led to a new therapeutic medicine, eribulin mesylate. ${ }^{7}$ In our continuous exploration of new chemical entities, we also designed and developed several additional series of novel anticancer agents according to this strategy. These agents include 2-(furan-2-yl) naphthalen-1-ol (FNO), ${ }^{8}$ 6-phenyl-4H-furo[3,2-c]pyran-4-one (AFPO), ${ }^{9}$ tetrahydronaphthalene-1-ol (TNO), 10 and 4-amino- $2 H$-benzo[ $h]$ chromen-2-one (ABO, 3 , Figure 1) ${ }^{11}$ analogs. Lead compounds showed potent antitumor activity and different tumor tissue type selectivity. Braccio et al first reported four compounds with the ABO scaffold and their cytotoxic activity against Ehrlich ascites tumor cells. ${ }^{12} \mathrm{We}$ also designed and explored this scaffold based on our studies of neo-tanshinlactone analogs $(\mathbf{1}, \mathbf{2})$. Compound $\mathbf{4}$ showed potent and broad antitumor activity compared with $\mathbf{1}$ and $\mathbf{2} .{ }^{11}$ Structure-activity relationship (SAR) studies on $\mathbf{3}$ indicated that (1) secondary amine $\left(\mathrm{R}^{2}\right.$ or $\left.\mathrm{R}^{3}=\mathrm{H}\right)$ is preferred over tertiary amine $\left(R^{2}\right.$ and $\left.R^{3} \neq H\right)$, (2) bulky groups are favored at $R^{2} / R^{3}$ position, (3) 3'-bromophenyl group can cause dramatic loss of potency, and (4) hydrogen is better than an ethyl group at $\mathrm{R}^{1}$ position. Our prior studies also suggested that a nonaromatic ring can greatly affect the antitumor activity and cancer cell line selectivity. ${ }^{10}$ Consequently, we have now designed scaffold $\mathbf{5}$ with a non-aromatic five- or six-membered A-ring (Figure 1). Different amino $\left(\mathrm{R}^{2} / \mathrm{R}^{3}\right)$ and A-ring $\left(\mathrm{R}^{1}\right)$ substituents were incorporated to establish SAR and identify potent analogs. This paper reports the design, synthesis, and biological activity of 4-amino-7,8,9,10-tetrahydro- $2 \mathrm{H}$-benzo $[h]$ chromen-2-one, (ATBO) analogs.

All target compounds 8-27, 32, and $\mathbf{3 3}$ were synthesized from the related 4-hydroxy compounds, $\mathbf{6}, \mathbf{2 8}$, and $\mathbf{2 9}$, respectively, according to the methods reported before (Scheme 1). Chlorides $\mathbf{7}, \mathbf{3 0}$, and $\mathbf{3 1}$ were synthesized by treatment of $\mathbf{6 , 2 8}$, and $\mathbf{2 9}$, respectively, with $\mathrm{POCl}_{3} .{ }^{11,12}$ Various amino derivatives, $\mathbf{8 - 2 7}, \mathbf{3 2}$, and 33, were newly prepared by substitution reactions of $\mathbf{7 , 3 0}$, and $\mathbf{3 1}$ with different amines according to reported procedures. ${ }^{12,13}$

All synthesized analogs 8-27 and 32-33 were tested for in vitro cytotoxic activity against a panel of human tumor cell lines according to previously published methods (Table 1).8, 14,15 Cell lines included A549 (non small cell lung cancer), DU145 (prostate cancer cell line), KB (nasopharyngeal carcinoma), KB-VIN (vincristine-resistant MDR KB subline), MDAMB-231 (estrogen receptor negative breast cancer), SK-BR-3 (estrogen receptor negative, HER2 over-expressing breast cancer), and ZR-75-1 (estrogen receptor positive breast cancer).

Regarding ATBO analogs with aliphatic $\mathrm{R}_{2} / \mathrm{R}_{3}$ amino substituents, $\mathbf{1 1}$ with a cyclohexyl group showed greater activity than the methyl, propyl, and cyclopropyl derivatives 8-10. Analog 10 with a cyclopropyl group displayed tumor cell line selectivity against KB, KBVIN, and ZR-75-1, while $\mathbf{8}$ and $\mathbf{9}$ showed greatly reduced or no activity against all tested cell lines. These findings suggested that a cyclic group is better than a linear group for cytotoxic activity, and a bulky amino group is favored. The tertiary amines $\mathbf{2 5 - 2 7}$ were not active, regardless of cyclic or linear amino groups; thus, a secondary amine is crucial for antitumor activity. The above results are consistent with our previous studies for $\mathrm{ABO}$ analogs. ${ }^{10}$ Interestingly, 8 and $\mathbf{9}$ showed unique selectivity against ZR-75-1 compared with 
other cell lines tested, although the inhibitory potency was only moderate. In addition, $\mathbf{1 1}$ with a cyclohexyl group showed similar potency to $\mathbf{1 2}$ with a phenyl group, which indicated that aromaticity of the amino substituent is not an essential factor.

Regarding aromatic $\mathrm{R}_{2} / \mathrm{R}_{3}$ substitutents, we explored different groups at the 2', 3', and 4' positions. Generally, all aromatic derivatives 12-24 showed significant cytotoxicity against the human tumor cell lines tested. Among them, $4^{\prime}-\mathrm{MeO} 15$ was the most potent analog against all tested tumor cell lines $\left(\mathrm{ED}_{50}\right.$ values of $\left.0.008-0.064 \mu \mathrm{M}\right)$. It was two- to three-fold more potent than the related ABO analog 4 against all cell lines, except ZR-75-1. Importantly, 15 also exhibited the highest cytotoxicity against MDA-MB-231 (ER negative breast cancer) compared with other cell lines. Analogs 10-13, 19, and 23-24 were less potent against MDA-MB-231 and 14, 16, 21-22 were less potent against both MDA-MB-231 and ZR-75-1 compared with other tested cell lines, which indicated different cancer cell line selectivity among ATBO analogs.

We further investigated the effect of ring-A. Analogs 15 and 32, with an unsubstituted six and five-membered ring, respectively, as well as analog 33, with a six-membered ring-A with gem-dimethyl substitution, displayed significant inhibition against all tested human tumor cell lines, but different selectivity against tumor cell lines from different tissues. Against KB, KB-VIN, A549, DU145, and SKBR-3, 15 and $\mathbf{3 3}$ were at least twofold more potent than 32. Against ZR-75-1 and MDA-MB-231, $15\left(\mathrm{ED}_{50} 0.024\right.$ and $0.008 \mu \mathrm{M}$, respectively) showed much higher activity as compared with $\mathbf{3 2}$ and $\mathbf{3 3}$. These results together with our previous findings indicated that a non-aromatic ring-A may lead to changes in both potency and cancer cell line selectivity of certain analogs. The size and identity of ring-A substituents are also important factors.

In conclusion, our structural modification and optimization resulted in a novel class of in vitro anti-cancer agents, ATBO. The results indicated that the non-aromatic ring-A of $\mathbf{5}$ is critical to both potency and cancer cell line selectivity. Based on this study, the preliminary SAR findings for the ATBO class were similar to those with ABO analogs. (1) Secondary amine $\left(\mathrm{R}_{2}\right.$ or $\left.\mathrm{R}_{3}=\mathrm{H}\right)$ is preferred over tertiary amine $\left(\mathrm{R}_{2}\right.$ and $\left.\mathrm{R}_{3} \neq \mathrm{H}\right),(2)$ bulky groups are favored at $R_{2} / R_{3}$ position, (3) aromaticity is not required at $R_{2} / R_{3}$ position, and (4) nonaromatic ring-A can increase potency and cancer cell line selectivity for certain analogs, such as 15. Compound 15 was the most potent analog $\left(\mathrm{ED}_{50}\right.$ values of $\left.0.008-0.064 \mu \mathrm{M}\right)$ among all $\mathrm{ABO}$ and $\mathrm{ATBO}$ derivatives, and thus, is a promising new lead compound for further development toward a potential clinical trials candidate.

\section{Acknowledgments}

This work was supported by NIH grant CA-17625 from the National Cancer Institute, awarded to K.H. Lee.

\section{References and notes}

1. Bleicher Konrad H, Bohm H-J, Muller K, Alanine Alexander I. Hit and lead generation: beyond high-throughput screening. Nat Rev Drug Discov 2003;2:369-78. [PubMed: 12750740]

2. Vallance P, Williams P, Dollery C. The future is much closer collaboration between the pharmaceutical industry and academic medical centers. Clin Pharmacol Ther 87:525-7. [PubMed: 20407455]

3. Hughes B. FDA drug approvals. Nat Rev Drug Discov 2009;9:89-92. [PubMed: 20118952]

4. Crommelin D, Stolk P, Besancon L, Shah V, Midha K, Leufkens H. Pharmaceutical sciences in 2020. Nat Rev Drug Discov 9:99-100. [PubMed: 20118959]

5. Wang X, Bastow KF, Sun CM, Lin YL, Yu HJ, Don MJ, Wu TS, Nakamura S, Lee KH. Antitumor Agents. 239. Isolation, structure elucidation, total synthesis, and anti-breast cancer activity of neotanshinlactone from Salvia miltiorrhiza. J Med Chem 2004;47:5816-9. [PubMed: 15509181] 
6. Wang X, Nakagawa-Goto K, Bastow KF, Don MJ, Lin YL, Wu TS, Lee KH. Antitumor agents. 254. Synthesis and biological evaluation of novel neo-tanshinlactone analogues as potent anti-breast cancer agents. J Med Chem 2006;49:5631-4. [PubMed: 16942038]

7. Kim DS, Dong CG, Kim JT, Guo H, Huang J, Tiseni PS, Kishi Y. New syntheses of E7389 C14C35 and halichondrin C14-C38 building blocks: double-inversion approach. J Am Chem Soc 2009;131:15636-41. [PubMed: 19807076]

8. Dong Y, Shi Q, Liu Y-N, Wang X, Bastow KF, Lee K-H. Antitumor Agents. 266. Design, Synthesis, and Biological Evaluation of Novel 2-(Furan-2-yl)naphthalen-1-ol Derivatives as Potent and Selective Antibreast Cancer Agents. J. Med. Chem 2009;52:3586-3590. [PubMed: 19425534]

9. Dong Y, Shi Q, Nakagawa-Goto K, Wu P-C, Morris-Natschke SL, Brossi A, Bastow KF, Lang J-Y, Hung M-C, Lee K-H. Antitumor agents 270. Novel substituted 6-phenyl-4H-furo[3,2-c]pyran-4-one derivatives as potent and highly selective anti-breast cancer agents. Bioorg. Med. Chem 18:803808. [PubMed: 20034799]

10. Dong Y, Shi Q, Nakagawa-Goto K, Wu PC, Bastow KF, Morris-Natschke SL, Lee KH. Antitumor agents 269. Non-aromatic ring-A neotanshinlactone analog, TNO, as a new class of potent antitumor agents. Bioorg Med Chem Lett 2009;19:6289-92. [PubMed: 19819136]

11. Yizhou Dong KN-G, Lai Chin-Yu, Morris-Natschke Susan L. Bastow Kenneth F. Lee KuoHsiung. Antitumor Agents 278. 4-Amino-2H-benzo[h]chromen-2-one (ABO) Analogs as Potent In Vitro Anticancer Agents. Bioorganic \& Medicinal Chemistry Letters. 2010 in press.

12. Di Braccio M, Grossi G, Roma G, Marzano C, Baccichetti F, Simonato M, Bordin F. Pyran derivatives. Part XXI. Antiproliferative and cytotoxic properties of novel N-substituted 4aminocoumarins, their benzo-fused derivatives, and some related 2-aminochromones. Farmaco 2003;58:1083-1097. [PubMed: 14572859]

13. Alberola A, Alvaro R, Ortega AG, Sadaba ML, Sanudo MC. Synthesis of [1]benzopyrano[4,3b]pyrrol-4(1H)-ones from N(alpha )-(2-oxo-2H-1-benzopyran-4-yl)weinreb alpha -aminoamides. Tetrahedron 1999;55:13211-13224.

14. Solubility of 12-14 and 16-18 in DMSO is not very great. Work is ongoing to solve the solubility issue and results will be reported in due course.

15. Spectroscopic data. 4-(methylamino)-7,8,9,10-tetrahydro- $2 H$-benzo[ $h]$ chromen-2-one $(8):{ }^{1} \mathrm{H}$ NMR (400 MHz, $\left.\mathrm{CDCl}_{3}\right): \delta 7.16(\mathrm{~d}, 1 \mathrm{H}, J=8.4 \mathrm{~Hz}, \mathrm{Ar}-H), 6.96(\mathrm{~d}, 1 \mathrm{H}, J=8.4 \mathrm{~Hz}, \mathrm{Ar}-H), 5.28$ $(\mathrm{s}, 2 \mathrm{H}, \mathrm{NH} \& 3-H), 3.00\left(\mathrm{~d}, 3 \mathrm{H}, J=4.8 \mathrm{~Hz}, \mathrm{NCH}_{3}\right), 2.90(\mathrm{t}, 2 \mathrm{H}, J=6.0 \mathrm{~Hz}, 10-H), 2.83(\mathrm{t}, 2 \mathrm{H}, J=$ $6.0 \mathrm{~Hz}, 7-H), 1.82$ (m, 4H, 8 \& 9-H). MS m/z 228 ( $\left.\mathrm{M}^{+}-1\right)$. 4-(propylamino)-7,8,9,10tetrahydro-2H-benzo[ $h]$ chromen-2-one (9): ${ }^{1} \mathrm{H} \mathrm{NMR}\left(400 \mathrm{MHz}, \mathrm{CDCl}_{3}\right): \delta 7.18(\mathrm{~d}, 1 \mathrm{H}, J=8.0$ $\mathrm{Hz}, \operatorname{Ar}-H), 6.96(\mathrm{~d}, 1 \mathrm{H}, J=8.0 \mathrm{~Hz}, \operatorname{Ar}-H), 5.28(\mathrm{~s}, 1 \mathrm{H}, 3-H), 5.19(\mathrm{br} \mathrm{s}, 1 \mathrm{H}, \mathrm{NH}), 3.22$ (q, 2H, $J=$ $\left.5.2 \mathrm{~Hz}, \mathrm{NCH}_{2}\right), 2.90(\mathrm{t}, 2 \mathrm{H}, J=6.0 \mathrm{~Hz}, 10-H), 2.82(\mathrm{t}, 2 \mathrm{H}, J=6.0 \mathrm{~Hz}, 7-H), 1.78(\mathrm{~m}, 6 \mathrm{H}, 8,9$ \& 2'- $H$ ), 1.04 (t, 3H, $J=7.2 \mathrm{~Hz}, 3$ '- $H$ ). MS $m / z 258$ ( $\left.\mathrm{M}^{+}+1\right)$. 4-(cyclopropylamino)-7,8,9,10tetrahydro-2H-benzo[ $h$ ]chromen-2-one (10): $\left.1 \mathrm{H} \mathrm{NMR} \mathrm{(400} \mathrm{MHz,} \mathrm{CDCl}_{3}\right): \delta 7.10(\mathrm{~d}, 1 \mathrm{H}, J=8.4$ $\mathrm{Hz}, \operatorname{Ar}-H), 6.94(\mathrm{~d}, 1 \mathrm{H}, J=8.0 \mathrm{~Hz}, \operatorname{Ar}-H), 5.72(\mathrm{~s}, 1 \mathrm{H}, 3-H), 5.41(\mathrm{br} \mathrm{s}, 1 \mathrm{H}, \mathrm{N} H), 2.90$ (t, 2H, $J=$ $5.6 \mathrm{~Hz}, 10-H), 2.83(\mathrm{t}, 2 \mathrm{H}, J=5.6 \mathrm{~Hz}, 7-H), 2.58(\mathrm{~m}, 1 \mathrm{H}, \mathrm{NCH}), 1.83(\mathrm{~m}, 4 \mathrm{H}, 8$ \& 9- $H), 0.91(\mathrm{dd}$ $\left.2 \mathrm{H}, J=5.6,5.2 \mathrm{~Hz}, \mathrm{NCHCH}_{2}\right), 0.68\left(\mathrm{dd}, 2 \mathrm{H}, J=5.2,4.4 \mathrm{~Hz}, \mathrm{NCHCH}_{2}\right) . \mathrm{MS} m / z 254\left(\mathrm{M}^{+}-1\right) .4-$ (cyclohexylamino)-7,8,9,10-tetrahydro-2 $H$-benzo[ $h$ ]chromen-2-one (11): ${ }^{1} \mathrm{H}$ NMR (400 MHz, $\left.\mathrm{CDCl}_{3}\right): \delta 7.15(\mathrm{~d}, 1 \mathrm{H}, J=8.0 \mathrm{~Hz}, \mathrm{Ar}-H), 6.95(\mathrm{~d}, 1 \mathrm{H}, J=8.4 \mathrm{~Hz}, \mathrm{Ar}-H), 5.31(\mathrm{~s}, 1 \mathrm{H}, 3-H), 5.01$ $(\mathrm{d}, 1 \mathrm{H}, J=5.6 \mathrm{~Hz}, \mathrm{NH}), 3.40\left(\mathrm{~m}, 1 \mathrm{H}, 1^{\prime}-H\right), 2.90(\mathrm{t}, 2 \mathrm{H}, J=6.0 \mathrm{~Hz}, 10-H), 2.81(\mathrm{t}, 2 \mathrm{H}, J=6.0$ $\mathrm{Hz}, 7-H), 2.12$ (m, 2H, 2' \& 6'-H), 1.78 (m, 6H, 8, 9 \& 2' \& 6'-H), 1.28 (m, 6H, 3' \& 4'\& 5'-H). MS m/z $298\left(\mathrm{M}^{+}+1\right)$. 4-phenylamino-7,8,9,10-tetrahydro-2H-benzo[h]chromen-2-one (12): ${ }^{1} \mathrm{H}$ NMR (400 MHz, DMSO-d 6 ): $\delta 9.20$ (br s, 1H, NH), 7.96 (d, 1H, J=8.2 Hz, Ar-H), 7.48 (dd, 1H, $J=8.2$ and $7.4 \mathrm{~Hz}, \mathrm{Ar}-H), 7.36(\mathrm{~d}, 1 \mathrm{H}, J=8.2 \mathrm{~Hz}, \mathrm{Ar}-H), 7.36(\mathrm{~d}, 1 \mathrm{H}, J=7.4 \mathrm{~Hz}, \mathrm{Ar}-H), 7.28$ $(\mathrm{dd}, 1 \mathrm{H}, J=7.4$ and $7.2 \mathrm{~Hz}, \mathrm{Ar}-H), 7.12(\mathrm{~d}, 1 \mathrm{H}, J=8.2 \mathrm{~Hz}, \mathrm{Ar}-H), 5.27$ (s, 1H, 3-H), 2.86-2.74 (m, 4H, 7 \& 10-H), 1.85-1.72 (m, 4H, 8 \& 9-H). MS m/z $292\left(\mathrm{M}^{+}+1\right)$. 4-[(4'methylphenyl)amino]-7,8,9,10-tetrahydro-2H-benzo[ $h]$ chromen-2-one (13): ${ }^{1} \mathrm{H}$ NMR $(400 \mathrm{MHz}$, DMSO-d $_{6}$ ): $\delta 9.14$ (br s, $\left.1 \mathrm{H}, \mathrm{N} H\right), 7.95(\mathrm{~d}, 1 \mathrm{H}, J=8.3 \mathrm{~Hz}, \mathrm{Ar}-H), 7.28(\mathrm{~d}, 2 \mathrm{H}, J=8.2 \mathrm{~Hz}, \operatorname{Ar}-H)$, 7.23 (d, $2 \mathrm{H}, J=8.2 \mathrm{~Hz}, \mathrm{Ar}-H), 5.19$ (s, $1 \mathrm{H}, 3-H), 2.86-2.74$ (m, 4H, 7 \& 10- $H$ ), 2.34 (s, 3H, Ar$\left.\mathrm{CH}_{3}\right), 1.84-1.71\left(\mathrm{~m}, 4 \mathrm{H}, 8\right.$ \& 9-H). MS m/z $306\left(\mathrm{M}^{+}+1\right)$. 4-[(4'-ethylphenyl)amino]-7,8,9,10tetrahydro-2 $H$-benzo[ $h$ ]chromen-2-one (14): ${ }^{1} \mathrm{H}$ NMR (400 MHz, DMSO-d 6 ): $\delta 9.14$ (br s, $1 \mathrm{H}$, $\mathrm{N} H), 7.95(\mathrm{~d}, 1 \mathrm{H}, J=8.4 \mathrm{~Hz}, \operatorname{Ar}-H), 7.31(\mathrm{~d}, 2 \mathrm{H}, J=8.4 \mathrm{~Hz}, \mathrm{Ar}-H), 7.25(\mathrm{~d}, 2 \mathrm{H}, J=8.4 \mathrm{~Hz}, \mathrm{Ar}-$ $H$ ), $7.11(\mathrm{~d}, 1 \mathrm{H}, J=8.4 \mathrm{~Hz}, \operatorname{Ar}-H), 5.20(\mathrm{~s}, 1 \mathrm{H}, 3-H), 2.86-2.73$ (m, 4H, 7 \& 10-H), 2.64 (q, $2 \mathrm{H}, J$ 
$\left.=7.5 \mathrm{~Hz}, \mathrm{Ar}-\mathrm{CH}_{2} \mathrm{CH}_{3}\right), 1.84-1.72(\mathrm{~m}, 4 \mathrm{H}, 8 \& 9-\mathrm{H}), 1.21\left(\mathrm{t}, 3 \mathrm{H}, \mathrm{J}=7.5 \mathrm{~Hz}, \mathrm{Ar}_{-} \mathrm{CH}_{2} \mathrm{CH}_{3}\right) . \mathrm{MS} \mathrm{m} /$ z $320\left(\mathrm{M}^{+}+1\right)$. 4-((4'-methoxyphenyl)amino)-7,8,9,10-tetrahydro-2 $H$-benzo[ $\left.h\right]$ chromen-2-one (15): ${ }^{1} \mathrm{H}$ NMR (400 MHz, DMSO-d 6 ): $\delta 9.09$ (br s, $\left.1 \mathrm{H}, \mathrm{NH}\right), 7.94(\mathrm{~d}, 1 \mathrm{H}, J=8.8 \mathrm{~Hz}, \mathrm{Ar}-H), 7.27$ $(\mathrm{d}, 2 \mathrm{H}, J=8.0 \mathrm{~Hz}, \mathrm{Ar}-H), 7.10(\mathrm{~d}, 1 \mathrm{H}, J=8.0 \mathrm{~Hz}, \mathrm{Ar}-H), 7.04(\mathrm{~d}, 2 \mathrm{H}, J=8.0 \mathrm{~Hz}, \mathrm{Ar}-H), 5.05(\mathrm{~s}$, $1 \mathrm{H}, 3-H), 3.79\left(\mathrm{~s}, 3 \mathrm{H}, \mathrm{OCH}_{3}\right), 2.82(\mathrm{t}, 2 \mathrm{H}, J=5.6 \mathrm{~Hz}, 10-H), 2.76(\mathrm{t}, 2 \mathrm{H}, J=5.6 \mathrm{~Hz}, 7-H), 1.78$ (m, 4H, 8 \& 9-H). MS m/z $320\left(\mathrm{M}^{+}-1\right)$. 4-[(4'-fluorophenyl)amino]-7,8,9,10-tetrahydro- $2 \mathrm{H}$ benzo[ $h]$ chromen-2-one (16): ${ }^{1} \mathrm{H}$ NMR (400 MHz, DMSO-d 6 ): $\delta 9.18$ (br s, $\left.1 \mathrm{H}, \mathrm{NH}\right), 7.93$ (d, $1 \mathrm{H}$, $J=8.2 \mathrm{~Hz}$, Ar- $H$ ), 7.42-7.36 (m, 2H, Ar- $H$ ), 7.35-7.28 (m, 2H, Ar- $H), 7.12(\mathrm{~d}, 1 \mathrm{H}, J=8.2 \mathrm{~Hz}, \mathrm{Ar}-$ $H$ ), 5.16 (s, 1H, 3-H), 2.86-2.73 (m, 4H, 7 \&10-H), 1.85-1.71 (m, 4H, 8 \& 9- $H$ ). MS m/z 310 $\left(\mathrm{M}^{+}+1\right)$. 4-[(4'-bromophenyl)amino]-7,8,9,10-tetrahydro-2H-benzo[ $\left.h\right]$ chromen-2-one (17): ${ }^{1} \mathrm{H}$ NMR (400 MHz, DMSO-d 6 ): $\delta 9.21($ br s, $1 \mathrm{H}, \mathrm{N} H), 7.92(\mathrm{~d}, 1 \mathrm{H}, J=8.3 \mathrm{~Hz}, \mathrm{Ar}-H), 7.68-7.62(\mathrm{~m}$, $2 \mathrm{H}, \operatorname{Ar}-H), 7.36-7.31(\mathrm{~m}, 2 \mathrm{H}, \mathrm{Ar}-H), 7.12(\mathrm{~d}, 1 \mathrm{H}, J=8.3 \mathrm{~Hz}, \mathrm{Ar}-H), 5.34$ (s, 1H, 3-H), 2.86-2.74 (m, 4H, $7 \& 10-H), 1.85-1.72\left(\mathrm{~m}, 4 \mathrm{H}, 8\right.$ \& 9-H). MS m/z 368 and $370\left(1: 1, \mathrm{M}^{+}-1\right)$. 4-[(2'methylphenyl)amino]-7,8,9,10-tetrahydro-2 $H$-benzo[ $h$ ]chromen-2-one (18): ${ }^{1} \mathrm{H}$ NMR $(400 \mathrm{MHz}$, DMSO-d $\left._{6}\right): \delta 9.10($ br s, $1 \mathrm{H}, \mathrm{N} H), 7.96(\mathrm{~d}, 1 \mathrm{H}, J=8.2 \mathrm{~Hz}, \mathrm{Ar}-H), 7.44-7.39(\mathrm{~m}, 1 \mathrm{H}, \mathrm{Ar}-H)$, 7.36-7.31 (m, 2H, Ar-H), 7.29-7.24 (m, $1 \mathrm{H}, \operatorname{Ar}-H), 7.12$ (d, 1H, $J=8.2 \mathrm{~Hz}, \operatorname{Ar}-H), 4.54$ (s, 1H, 3$H$ ), 2.86-2.74 (m, 4H, 7 \&10-H), 2.18 (s, 3H, Ar-CH3), 1.85-1.72 (m, 4H, 8 \& 9-H). MS m/z 306 $\left(\mathrm{M}^{+}+1\right)$. 4-[(2'-methoxyphenyl)amino]-7,8,9,10-tetrahydro-2H-benzo[ $\left.h\right]$ chromen-2-one (19): ${ }^{1} \mathrm{H}$ NMR (400 MHz, DMSO-d 6 ): $\delta 8.90($ br s, $1 \mathrm{H}, \mathrm{NH}), 7.96(\mathrm{~d}, 1 \mathrm{H}, J=8.2 \mathrm{~Hz}, \mathrm{Ar}-H), 7.42-7.34(\mathrm{~m}$, $1 \mathrm{H}, \operatorname{Ar}-H), 7.32-7.26(\mathrm{~m}, 1 \mathrm{H}, \operatorname{Ar}-H), 7.20(\mathrm{~d}, 1 \mathrm{H}, J=8.2 \mathrm{~Hz}, \operatorname{Ar}-H), 7.10(\mathrm{~d}, 1 \mathrm{H}, J=8.2 \mathrm{~Hz}, \mathrm{Ar}-$ $H$ ), 7.09-7.05 (m, 1H, Ar- $H$ ), $4.68(\mathrm{~s}, 1 \mathrm{H}, 3-H), 3.79$ (s, 3H, OCH $), 2.96-2.72(\mathrm{~m}, 4 \mathrm{H}, 7$ \& $10-H)$, 1.86-1.70 (m, 4H, 8 \& 9-H). MS m/z $322\left(\mathrm{M}^{+}+1\right)$. 4-[(2'-bromophenyl)amino]-7,8,9,10tetrahydro-2 $H$-benzo[ $h$ ]chromen-2-one (20): $1 \mathrm{H} \mathrm{NMR}\left(400 \mathrm{MHz}, \mathrm{DMSO}-\mathrm{d}_{6}\right): \delta 9.21$ (br s, $1 \mathrm{H}$, $\mathrm{N} H)$, 7.98-7.90 (m, 1H, Ar-H), 7.70-7.62 (m, 1H, Ar-H), 7.50-7.24 (m, 3H, Ar-H), 7.12 (d, $1 \mathrm{H}, J$ $=8.3 \mathrm{~Hz}, \operatorname{Ar}-H), 5.35(\mathrm{~s}, 1 \mathrm{H}, 3-H), 2.86-2.74(\mathrm{~m}, 4 \mathrm{H}, 7 \& 10-H), 1.85-1.72(\mathrm{~m}, 4 \mathrm{H}, 8 \& 9-H)$. MS $m / z 368$ and $370\left(1: 1, \mathbf{M}^{+}-1\right)$. 4-[(3'-methylphenyl)amino]-7,8,9,10-tetrahydro- $2 H$ -

benzo[ $h]$ chromen-2-one (21): ${ }^{1} \mathrm{H}$ NMR (400 MHz, DMSO-d 6 ): $\delta 9.15$ (br s, $\left.1 \mathrm{H}, \mathrm{NH}\right), 7.95$ (d, $1 \mathrm{H}$, $J=8.3 \mathrm{~Hz}, \operatorname{Ar}-H), 7.36(\mathrm{t}, 1 \mathrm{H}, J=7.7 \mathrm{~Hz}, \operatorname{Ar}-H), 7.19-7.08(\mathrm{~m}, 3 \mathrm{H}, \operatorname{Ar}-H), 5.26(\mathrm{~s}, 1 \mathrm{H}, 3-H)$, 2.86-2.74 (m, 4H, 7 \&10-H), 2.36 (s, 3H, Ar-CH $\left.H_{3}\right), 1.85-1.72(\mathrm{~m}, 4 \mathrm{H}, 8$ \& 9- $H$ ). MS m/z 306 $\left(\mathrm{M}^{+}+1\right)$. 4-[(3'-methoxyphenyl)amino]-7,8,9,10-tetrahydro-2H-benzo[ $\left.h\right]$ chromen-2-one $(22):{ }^{1} \mathrm{H}$ NMR (400 MHz, DMSO-d 6 ): $\delta 9.16$ (br s, 1H, NH), 7.94 (d, 1H, $J=8.4 \mathrm{~Hz}, \mathrm{Ar}-H), 7.38$ (t, $1 \mathrm{H}, J$ $=8.1 \mathrm{~Hz}, \mathrm{Ar}-H), 7.11(\mathrm{~d}, 1 \mathrm{H}, J=8.4 \mathrm{~Hz}, \mathrm{Ar}-H), 6.98-6.92(\mathrm{~m}, 1 \mathrm{H}, \mathrm{Ar}-H), 6.92-6.89(\mathrm{~m}, 1 \mathrm{H}, \mathrm{Ar}-$ $H), 6.85(\mathrm{dd}, 1 \mathrm{H}, J=8.1$ and $2.5 \mathrm{~Hz}, \mathrm{Ar}-H), 5.35(\mathrm{~s}, 1 \mathrm{H}, 3-H), 3.79\left(\mathrm{~s}, 3 \mathrm{H}, \mathrm{OCH}_{3}\right), 2.86-2.74(\mathrm{~m}$, 4H, $7 \& 10-H), 1.85-1.72\left(\mathrm{~m}, 4 \mathrm{H}, 8\right.$ \& 9-H). MS m/z $322\left(\mathrm{M}^{+}+1\right)$. 4-[(3'-

bromophenyl)amino]-7,8,9,10-tetrahydro-2 $H$-benzo[ $h]$ chromen-2-one (23): ${ }^{1} \mathrm{H}$ NMR (400 MHz, DMSO-d 6 ): $\delta 9.24$ (br s, $1 \mathrm{H}, \mathrm{N} H), 7.91(\mathrm{~d}, 1 \mathrm{H}, J=8.2 \mathrm{~Hz}, \mathrm{Ar}-H), 7.48-7.59-7.56(\mathrm{~m}, 1 \mathrm{H}, \mathrm{Ar}-H)$, $7.40(\mathrm{~m}, 3 \mathrm{H}, \mathrm{Ar}-H), 7.13$ (d, $1 \mathrm{H}, J=8.2 \mathrm{~Hz}, \mathrm{Ar}-H), 5.39$ (s, 1H, 3-H), 2.86-2.74 (m, 4H, 7 \&10$H$ ), 1.85-1.72 (m, 4H, 8 \& 9-H). MS m/z 368 and $370\left(1: 1, \mathrm{M}^{+}-1\right)$. 4-(naphthalen-1ylamino)-7,8,9,10-tetrahydro- $2 H$-benzo[ $h]$ chromen-2-one (24): ${ }^{1} \mathrm{H}$ NMR (400 MHz, DMSO-d 6 ): $\delta 9.54$ (br s, $1 \mathrm{H}, \mathrm{N} H), 8.13$ (d, $1 \mathrm{H}, J=8.2 \mathrm{~Hz}, \operatorname{Ar}-H), 8.05$ (d, $1 \mathrm{H}, J=7.4 \mathrm{~Hz}, \operatorname{Ar}-H), 8.01$ (d, $1 \mathrm{H}$, $J=8.2 \mathrm{~Hz}, \mathrm{Ar}-H), 7.81(\mathrm{~d}, 1 \mathrm{H}, J=8.2 \mathrm{~Hz}, \mathrm{Ar}-H), 7.65(\mathrm{dd}, 1 \mathrm{H}, J=8.2$ and $7.4 \mathrm{~Hz}, \mathrm{Ar}-H)$, 7.64-7.53 (m, 3H, Ar- $H$ ), 7.18 (d, 1H, $J=8.2 \mathrm{~Hz}, \mathrm{Ar}-H), 4.50$ (s, 1H, 3-H), 2.90-2.75 (m, 4H, 7 \&10- $H$ ), 1.86-1.74 (m, 4H, 8 \& 9-H) . MS m/z $342\left(\mathrm{M}^{+}+1\right)$. 4-(dipropylamino)-7,8,9,10tetrahydro-2H-benzo[ $h]$ chromen-2-one (25): $\left.{ }^{1} \mathrm{H} \mathrm{NMR} \mathrm{(400} \mathrm{MHz,} \mathrm{CDCl}_{3}\right): \delta 7.37(\mathrm{~d}, 1 \mathrm{H}, J=8.4$ $\mathrm{Hz}, \mathrm{Ar}-H), 6.94(\mathrm{~d}, 1 \mathrm{H}, J=8.4 \mathrm{~Hz}, \mathrm{Ar}-H), 5.59$ (s, $1 \mathrm{H}, 3-H), 3.27$ (t, $\left.4 \mathrm{H}, J=6.0 \mathrm{~Hz}, \mathrm{~N}\left(\mathrm{CH}_{2}\right)_{2}\right)$, $2.91(\mathrm{t}, 2 \mathrm{H}, J=5.6 \mathrm{~Hz}, 10-H), 2.82(\mathrm{t}, 2 \mathrm{H}, J=5.6 \mathrm{~Hz}, 7-H), 1.83(\mathrm{~m}, 4 \mathrm{H}, 8$ \& 9-H), $1.68(\mathrm{~m}, 4 \mathrm{H}$, $\left.\mathrm{N}\left(\mathrm{CH}_{2} \mathrm{CH}_{2} \mathrm{CH}_{3}\right)_{2}\right), 0.90\left(\mathrm{t}, 6 \mathrm{H}, J=7.6 \mathrm{~Hz}, \mathrm{~N}\left(\mathrm{CH}_{2} \mathrm{CH}_{2} \mathrm{CH}_{3}\right)_{2}\right)$. MS m/z $300\left(\mathrm{M}^{+}+1\right) .4-$ (piperidin-1-yl)-7,8,9,10-tetrahydro- $2 H$-benzo[h]chromen-2-one (26): ${ }^{1} \mathrm{H}$ NMR (400 MHz, $\left.\mathrm{CDCl}_{3}\right): \delta 7.32(\mathrm{~d}, 1 \mathrm{H}, J=8.0 \mathrm{~Hz}, \mathrm{Ar}-H), 6.94(\mathrm{~d}, 1 \mathrm{H}, J=8.4 \mathrm{~Hz}, \mathrm{Ar}-H), 5.64(\mathrm{~s}, 1 \mathrm{H}, 3-H), 3.20$ $\left(\mathrm{t}, 4 \mathrm{H}, J=5.6 \mathrm{~Hz}, \mathrm{~N}\left(\mathrm{CH}_{2}\right)_{2}\right), 2.90(\mathrm{t}, 2 \mathrm{H}, J=5.6 \mathrm{~Hz}, 10-H), 2.82(\mathrm{t}, 2 \mathrm{H}, J=5.6 \mathrm{~Hz}, 7-H), 1.81$ $(\mathrm{m}, 8 \mathrm{H}, 8$ \& 9 \& 3' \& 5'- $\mathrm{H}), 1.72\left(\mathrm{~m}, 2 \mathrm{H}, \mathrm{NCH}_{2} \mathrm{CH}_{2} \mathrm{CH}_{2}\right)$. MS m/z $284\left(\mathrm{M}^{+}+1\right) .4-$ morpholino-7,8,9,10-tetrahydro-2 $H$-benzo[ $h]$ chromen-2-one (27): ${ }^{1} \mathrm{H} \mathrm{NMR}\left(400 \mathrm{MHz}, \mathrm{CDCl}_{3}\right): \delta$ $7.32(\mathrm{~d}, 1 \mathrm{H}, J=8.4 \mathrm{~Hz}, \operatorname{Ar}-H), 6.96(\mathrm{~d}, 1 \mathrm{H}, J=8.4 \mathrm{~Hz}, \mathrm{Ar}-H), 5.69(\mathrm{~s}, 1 \mathrm{H}, 3-H), 3.92(\mathrm{t}, 4 \mathrm{H}, J=$ $\left.4.8 \mathrm{~Hz}, \mathrm{O}\left(\mathrm{CH}_{2}\right)_{2}\right), 3.23\left(\mathrm{t}, 4 \mathrm{H}, J=4.8 \mathrm{~Hz}, \mathrm{~N}\left(\mathrm{CH}_{2}\right)_{2}\right), 2.90(\mathrm{t}, 2 \mathrm{H}, J=5.6 \mathrm{~Hz}, 10-H), 2.83(\mathrm{t}, 2 \mathrm{H}$, $J=5.6 \mathrm{~Hz}, 7-H), 1.83(\mathrm{~m}, 4 \mathrm{H}, 8 \&$ 9- $H)$. MS m/z $286\left(\mathrm{M}^{+}+1\right)$. 4-((4'-methoxyphenyl)amino)-8,9dihydrocyclopenta[$h]$ chromen-2(7H)-one (32): ${ }^{1} \mathrm{H}$ NMR (400 MHz, DMSO-d 6 ): $\delta 9.13$ (br s, $1 \mathrm{H}$, $\mathrm{N} H), 8.00(\mathrm{~d}, 1 \mathrm{H}, J=8.0 \mathrm{~Hz}, \operatorname{Ar}-H), 7.27$ (m, 3H, Ar- $H), 7.04$ (d, 2H, $J=8.4 \mathrm{~Hz}, \mathrm{Ar}-H), 5.04$ (s, 
1H, 3-H), 3.79 (s, 3H, $\left.\mathrm{OCH}_{3}\right), 3.00(\mathrm{~m}, 4 \mathrm{H}, 7$ \& 9- $H$ ), 2.13 (m, 2H, 8- $H)$. MS m/z $306\left(\mathrm{M}^{+}-1\right)$. 4((4'-methoxyphenyl)amino)-7,7-dimethyl-7,8,9,10-tetrahydro- $2 H$-benzo[ $h]$ chromen-2-one (33): ${ }^{1} \mathrm{H}$ NMR (400 MHz, DMSO-d 6 ): $\delta 9.10$ (br s, $\left.1 \mathrm{H}, \mathrm{NH}\right), 8.00(\mathrm{~d}, 1 \mathrm{H}, J=8.4 \mathrm{~Hz}, \mathrm{Ar}-H), 7.41$ $(\mathrm{d}, 1 \mathrm{H}, J=8.4 \mathrm{~Hz}, \mathrm{Ar}-H), 7.25$ (d, 2H, $J=8.4 \mathrm{~Hz}, \mathrm{Ar}-H), 7.04$ (d, 2H, $J=8.8 \mathrm{~Hz}, \operatorname{Ar}-H), 5.04$ (s, $1 \mathrm{H}, 3-H), 3.79\left(\mathrm{~s}, 3 \mathrm{H}, \mathrm{OCH}_{3}\right), 2.76(\mathrm{t}, 2 \mathrm{H}, J=6.0 \mathrm{~Hz}, 10-H), 1.79(\mathrm{~m}, 2 \mathrm{H}, 9-H), 1.65(\mathrm{~m}, 2 \mathrm{H}, 8-$ $H), 1.30\left(\mathrm{~s}, 6 \mathrm{H},\left(\mathrm{CH}_{3}\right)_{2}\right)$. MS m/z $348\left(\mathrm{M}^{+}-1\right)$. 


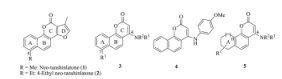

Figure 1.

Structures of neo-tanshinlactone (1), 4-ethyl neo-tanshinlactone (2), previously reported scaffold $\mathbf{3}$ and its analog $\mathbf{4}$, and newly designed scaffold $\mathbf{5}$ 


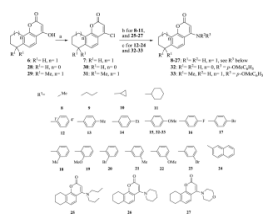

Scheme 1.

Reagents and conditions: (a) $\mathrm{POCl}_{3}, \mathrm{Et}_{3} \mathrm{~N}$, reflux, 1h; (b) aliphatic amines, EtOH, reflux, 2h; (c) aromatic amines, ethylene glycol, $160^{\circ} \mathrm{C}, 1 \mathrm{~h}$. 
